\title{
Signal processing for plane wave actuators
}

\author{
T. Corbach, M. Holters, and U. Zölzer \\ Helmut-Schmidt-University, University of the Federal Armed Forces Hamburg, Germany
}

\begin{abstract}
Plane wave actuators without an enclosure per se have a forward and backward radiation. The backward radiation is unwanted in many applications when a single direction radiation is desired. To avoid the disadvantages of an enclosure a system is proposed, which provides a high suppression of the unwanted backward radiation using a pair of plane wave actuators. This is achieved by adapted input signal filters. The influences of the second plane wave actuator to the forward radiated signal are suppressed as well. Additionally, the system also provides for- and backward radiation of different signals with a high suppression of the radiation directions crosstalk. The required power for the signal suppression depends on the physical damping of the plane wave actuators and the space in between. The first realized prototype is designed for flat panel dipole loudspeakers to deal with the mentioned problems in the acoustic domain. The filter design and a calibration algorithm for any given pairs of dipole loudspeaker are explained. The good performance of the developed system is proven by measurement results with the prototype system.
\end{abstract}

\section{Introduction}

Directed radiation is wanted in many applications to lower the effect of interference or to concentrate the radiated intensity to the target area. Most point source actuators per se radiate omnidirectional, whereas actuators of nearly plane waves mainly radiate to the forward and backward. Approximating a plane wave actuator by a two-dimensional array of point sources achieves similar directivity including the backward radiation. The backward radiation of both, plane wave actuators and its point source approximation, is unwanted in

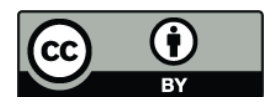

Correspondence to: T. Corbach (corbach@hsu-hh.de) many applications when a single direction radiation is desired. However, an additional enclosure effectively suppressing the backward radiation increases the size and hence lowers the good structural integration properties. The first realized prototype is designed for electrostatic flat panel dipole loudspeakers to deal with the mentioned problems in the acoustic domain. This paper presents alternative approaches to achieve a one sided radiation, describes the theory of the developed novel approach, demonstrates a practical realization and shows the results achieved with a DSP-based prototype.

\section{Alternative approaches}

Different approaches to suppress acoustical or electromagnetic traveling waves are available. Regardless the physical domain one cancellation method suppressing backward travelling wave is to use a $180^{\circ}$ phase delayed second wave. The destructive superposition leads to a cancellation.

To suppress the backward radiation of an electrostatic flat panel loudspeaker by active measures a second parallel similar panel is used. A $180^{\circ}$ phase delay and an additional delay according to the panel distance cancels out one radiation direction (Olson, 1973). This approach only grants the suppression of the backward radiation but does not take the influences on the forward radiation into account. Due to the dipole character of both panels the forward radiation is inevitably disturbed by the signal of the second panel.

A further approach of suppressing the backward radiation is the use of passive measures like an enclosure. But a high degree of suppression with passive measures is achieved at the cost of the structural integration properties of the radiator. Furthermore, the enclosure itself causes reflections disturbing the forward radiation. It has to be noted that these reflections can be suppressed with the proposed approach as well. 


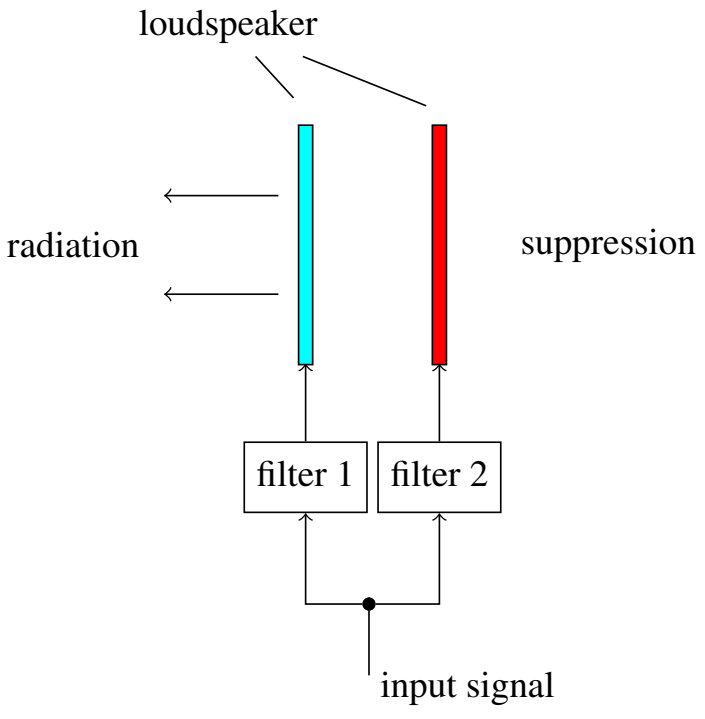

Fig. 1. Scheme of the proposed system with the desired radiation.

\section{Functionality}

In this section the theory and functionality of the single-sided radiation is explained first, followed by a short explanation of the mentioned stereo expansion. Unlike the alternative approaches the developed system is able to treat forward and backward radiation as independent channels with a low crosstalk.

The proposed system is based on a parallel double electrostatic dipole loudspeaker with a digital signal processing of the input signal. The desired digital filters are gained by transfer functions of the system which have to be determined. These transfer functions represent the behavior of every single subsystem which the system can be divided into. Once the subsystems are described and determined it is possible to compute the desired filter transfer functions as well as the filter impulse responses in time domain (Corbach et al., 2009).

\subsection{Subsystem transfer function}

The system is divided into subsystems according to the active panel $s \in\{1,2\}$ controlled by the discrete input signal $x(n)$ and the occurring discrete output signal $y_{s k}(n)$ at a reference position at the observed radiation direction $k \in\{l, r\}$. Due to the assumption of plane waves one reference position on axis is sufficient. For 2 panels and 2 possible radiation directions of the whole system, there is a total of 4 subsystems as depicted in Fig. 2.

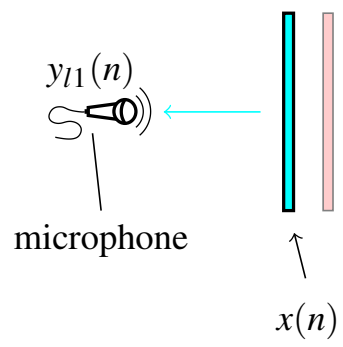

(a) subsystem 1

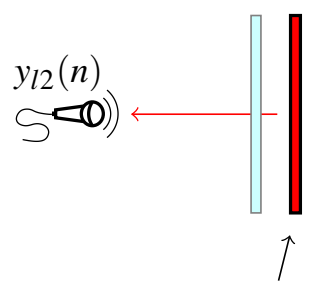

$x(n)$

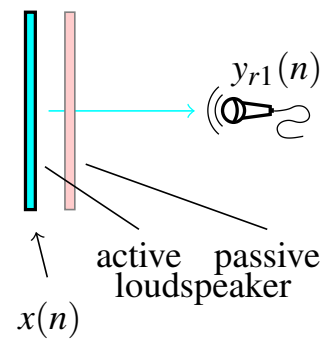

(b) subsystem 2

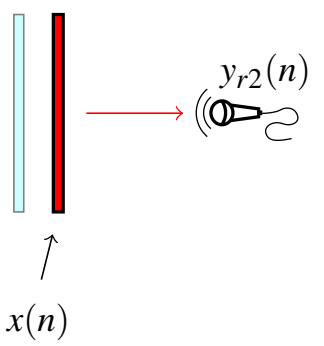

(d) subsystem 4

\section{(c) subsystem 3}

Fig. 2. Partition of the system into its subsystems (a) to (d) according the actuated panel and the observed side of the system.

In time domain the occurring output signals of the subsystems are

$$
\begin{aligned}
& y_{l 1}(n)=h_{l 1}(n) \cdot x(n), \\
& y_{r 1}(n)=h_{r 1}(n) \cdot x(n), \\
& y_{l 2}(n)=h_{l 2}(n) \cdot x(n), \text { and } \\
& y_{r 2}(n)=h_{r 2}(n) \cdot x(n) .
\end{aligned}
$$

For example the impulse response $h_{l 1}(n)$ of subsystem 1 (Fig. 2a) describes all influences to the input signal $x(n)$ radiated by loudspeaker 1 and the output signal $y_{l 1}(n)$ is observed on the left side of the double dipole. The impulse response $h_{r 1}(n)$ (Fig. 2b) describes all influences to the input signal $x(n)$ radiated by the same loudspeaker and the output signal $y_{r 1}(n)$ is observed on the opposite side of the double dipole. All reflections and transmission through the passive and active panels are included in the particular impulse response.

It has to be noted that this special case of a symmetric configuration of similar panels leads to equal impulse responses for the subsystems 1 and 4 (Fig. 2a and d), as well as for the subsystems 2 and 3 (Fig. $2 b$ and c). To keep the filter computation general this simplification is not done here.

The subsystem transfer functions can be determined by common measuring techniques like swept sine or noise sequences. Here the logarithmic swept sine technique (Farina, 2007 ) is used. The desired subsystem impulse responses are 
defined by

$h_{l 1}(N-n)=y_{l 1}(n) \cdot x_{\mathrm{swp}}^{-1}(n)$,

$h_{r 1}(N-n)=y_{r 1}(n) \cdot x_{\mathrm{swp}}^{-1}(n)$,

$h_{l 2}(N-n)=y_{l 2}(n) \cdot x_{\mathrm{swp}}^{-1}(n)$, and

$h_{r 2}(N-n)=y_{r 2}(n) \cdot x_{\mathrm{swp}}^{-1}(n)$,

where $x_{\mathrm{swp}}(n)$ denotes the sweep test signal, $x_{\mathrm{swp}}^{-1}(n)$ denotes the inverse sweep and $y_{s, k}(n)$ the measured sequence at the observed side. The test signal $x_{\mathrm{swp}}(n)$ and its inverse $x_{\mathrm{swp}}^{-1}(n)$ are both of length $N$.

As the measurements take place at just one point, the functionality of the system is guaranteed in exactly this measuring point. The high directivity and the low distance between the panels enlarge the area of controlled radiation nearly to the full sphere.

\subsection{Filter impulse response}

To determine the desired filter impulse responses the whole system is described by the subsystems' impulse responses. This is achieved by expressing all appearing signal parts by the input signal, the desired filter impulse response, and the subsystems' impulse responses. The signal part on the left side, created by input signal $x_{\text {in }}(n)$ convolved with the unknown filter impulse response $h_{f 1}(n)$ and the impulse response $h_{l 1}$ of subsystem 1 , can be denoted by

$x_{l 1}(n)=h_{l 1}(n) \cdot \underbrace{h_{f 1}(n) \cdot x_{\text {in }}(n)}_{x_{1}(n)}$.

Similarly, the other signal parts on both radiation sides of the double dipole loudspeaker can be denoted by

$$
\begin{aligned}
& x_{r 1}(n)=h_{r 1}(n) \cdot \underbrace{h_{f 1}(n) \cdot x_{\mathrm{in}}(n)}_{x_{1}(n)}, \\
& x_{l 2}(n)=h_{l 2}(n) \cdot \underbrace{h_{f 2}(n) \cdot x_{\mathrm{in}}(n)}_{x_{2}(n)} \text { and } \\
& x_{r 2}(n)=h_{r 2}(n) \cdot \underbrace{h_{f 2}(n) \cdot x_{\mathrm{in}}(n)}_{x_{2}(n)} .
\end{aligned}
$$

In the further equations the substitutions

$$
\begin{aligned}
& x_{1}(n)=h_{f 1}(n) \cdot x_{\mathrm{in}}(n) \text { and } \\
& x_{2}(n)=h_{f 2}(n) \cdot x_{\mathrm{in}}(n)
\end{aligned}
$$

are done.

Figure 3 shows the whole system with its signal parts $x_{l 1}(n), x_{r 1}(n), x_{l 2}(n)$ and $x_{r 2}(n)$ of the different subsystems. To realize a single-sided radiation by the filtered excitation, the signal parts have to result in the behavior of a single panel

$x_{l 1}(n)+x_{l 2}(n)=h_{l 1}(n) \cdot x_{\mathrm{in}}(n)$

for the desired radiation side.
To achieve the suppression of the second, unwanted radiation direction the filtered excitation has to result in signal parts cancelling out each other. By equation this yields

$x_{r 1}(n)+x_{r 2}(n)=0$.

Inserting Eqs. (9) and (11) into Eq. (15), and Eqs. (10) and (12) into Eq. (16) results in the required equations to describe the desired radiation characteristic by the subsystems and its signal parts:

$$
\begin{aligned}
& h_{l 1}(n) \cdot x_{1}(n)+h_{l 2}(n) \cdot x_{2}(n)=h_{l 1}(n) \cdot x_{\mathrm{in}}(n) \\
& h_{r 1}(n) \cdot x_{1}(n)+h_{r 2}(n) \cdot x_{2}(n) \quad=0 .
\end{aligned}
$$

The final computation of the filter impulse responses $h_{f 1}(n)$ and $h_{f 2}(n)$ is done in the frequency domain. Therefore the time signals of Eqs. (17) and (18) are transformed into frequency domain by the Fourier transform with adequate time domain zero padding:

$$
\begin{aligned}
H_{l 1}\left(e^{j \Omega}\right) X_{1}\left(e^{j \Omega}\right)+H_{l 2}\left(e^{j \Omega}\right) X_{2}\left(e^{j \Omega}\right) & =H_{l 1}\left(e^{j \Omega}\right) X_{\text {in }}\left(e^{j \Omega}\right)(19) \\
H_{r 1}\left(e^{j \Omega}\right) X_{1}\left(e^{j \Omega}\right)+H_{r 2}\left(e^{j \Omega}\right) X_{2}\left(e^{j \Omega}\right) & =0 .
\end{aligned}
$$

The back substitutions in frequency domain

$$
\begin{aligned}
& X_{1}\left(e^{j \Omega}\right)=H_{f 1}\left(e^{j \Omega}\right) X_{\text {in }}\left(e^{j \Omega}\right) \text { and } \\
& X_{2}\left(e^{j \Omega}\right)=H_{f 2}\left(e^{j \Omega}\right) X_{\text {in }}\left(e^{j \Omega}\right),
\end{aligned}
$$

as well as solving the Eqs. (19) and (20) for the filter transfer functions $H_{f 1}\left(e^{j \Omega}\right)$ and $H_{f 2}\left(e^{j \Omega}\right)$ gives

$$
\begin{aligned}
& H_{f 1}\left(e^{j \Omega}\right)=\frac{H_{l 1}\left(e^{j \Omega}\right) H_{r 2}\left(e^{j \Omega}\right)}{H_{l 1}\left(e^{j \Omega}\right) H_{r 2}\left(e^{j \Omega}\right)-H_{r 1}\left(e^{j \Omega}\right) H_{l 2}\left(e^{j \Omega}\right)} \text { and } \\
& H_{f 2}\left(e^{j \Omega}\right)=\frac{-H_{r 1}\left(e^{j \Omega}\right)}{H_{r 2}\left(e^{j \Omega}\right)} H_{f 1}\left(e^{j \Omega}\right) .
\end{aligned}
$$

Finally the inverse DFT is applied to the filter transfer functions to obtain the desired filter impulse responses $h_{f 1}(n)$ and $h_{f 2}(n)$ :

$H_{f 1}\left(e^{j \Omega}\right) \bullet \multimap h_{f 1}(n) \quad H_{f 2}\left(e^{j \Omega}\right) \bullet \multimap h_{f 2}(n)$.

\subsection{Signal computation}

The filtered input signals $x_{f 1}(n)$ and $x_{f 2}(n)$ actuating the panels are computed by a linear convolution with the corresponding filter impulse response $h_{f 1}(n)$ respectively $h_{f 2}(n)$ :

$x_{f 1}(n)=h_{f 1}(n) \cdot x_{\text {in }}(n)$
$x_{f 2}(n)=h_{f 2}(n) \cdot x_{\text {in }}(n)$

Depending on the given setup of loudspeakers and its distances, the filter transfer functions may show gains of several $\mathrm{dB}$ for certain frequencies. For input signals with amplitudes using the full quantization range, clipping will occur leading to worse performance and decreased audio quality. To avoid this, the filter transfer function has to be normalized to $0 \mathrm{~dB}$ or an equivalent damping factor should be introduced in the time domain. 


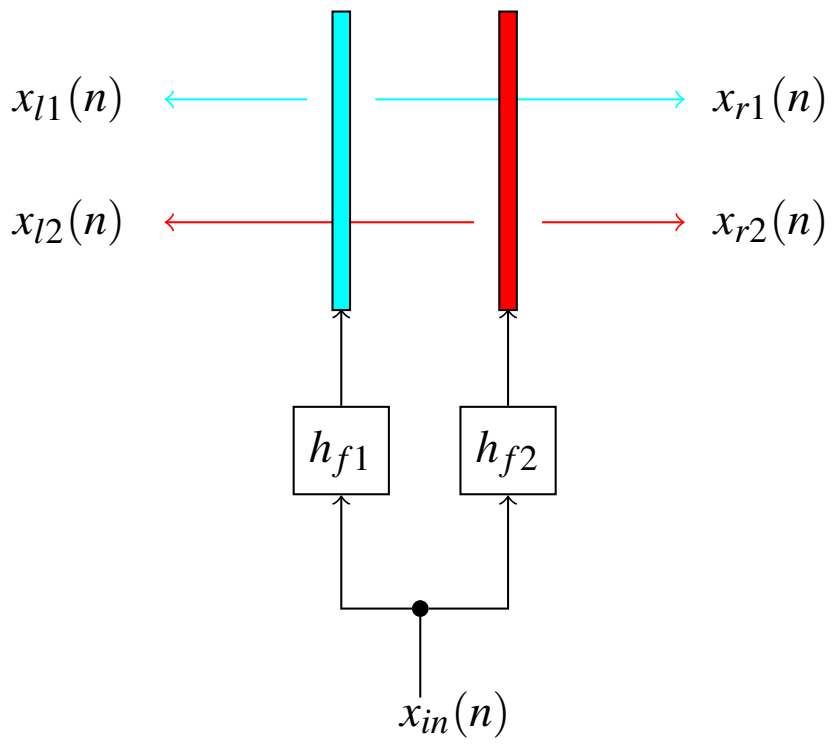

Fig. 3. Scheme of the entire system including all signal parts radiated by the single panels.

\subsection{Synthetic filter impulse response}

To clarify the systems functionality a filter computation with the use of synthetic subsystem impulse responses is done. Therefore, the plane wave sources are assumed to be ideal and equal at a sample-distance of $d$, and the wave transmission through the source is assumed to have a frequency independent attenuation of $g$.

Due to the symmetry this leads to equal impulse responses

$h_{d}(n)=h_{l 1}(n)=h_{r 2}(n)=\delta\left(n-d_{d}\right)$

for the subsystems 1 and 4, with $d_{d}$ representing the samples needed from the source to the reference position. Since the subsystems 2 and 3 describe the transmission through a source to the reference point, a $d_{t}$-delayed and attenuated unit impulse yields their impulse response

$h_{t}(n)=h_{l 2}(n)=h_{r 1}(n)=g \cdot \delta\left(n-d_{t}\right)$.

With direct delay of $d_{d}=5$, transmission delay of $d_{t}=9$, and an attenuation of $g=0.8$ the subsystems are described by the impulse responses $h_{d}(n)$ and $h_{t}(n)$ depicted in Figs. 4 and 5.

The Fourier transform yields the subsystems' transfer function $H_{l 1}\left(e^{j \Omega}\right), H_{r 2}\left(e^{j \Omega}\right), H_{l 2}\left(e^{j \Omega}\right)$, and $H_{r 1}\left(e^{j \Omega}\right)$. The desired filter transfer functions $H_{f 1}\left(e^{j \Omega}\right)$ and $H_{f 2}\left(e^{j \Omega}\right)$ are computed using Eqs. (23) and (24). The inverse Fourier transform then gives the desired filter impulse responses $h_{f 1}(n)$ and $h_{f 2}(n)$ depicted in Figs. 6 and 7. The sampledistance $d$ between each impulse in the filter impulse response exactly amounts

$d=d_{t}-d_{d}=9-5=4$,

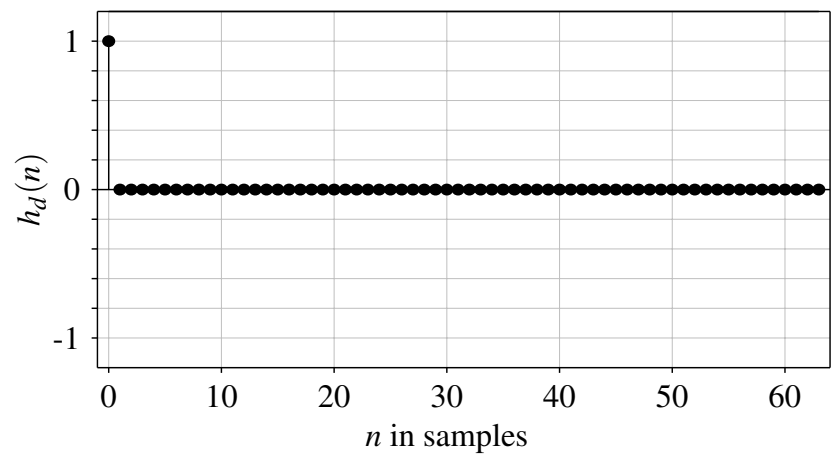

Fig. 4. Synthetic impulse response of the sub systems 1 and 4.

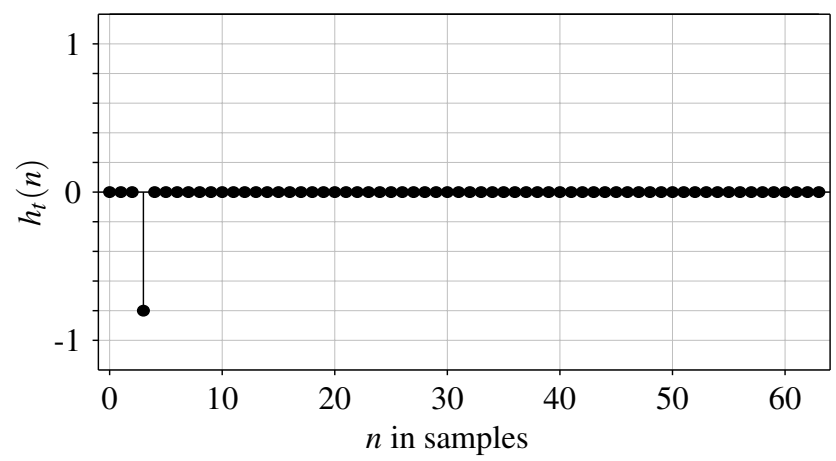

Fig. 5. Synthetic impulse response of the sub systems 2 and 3.

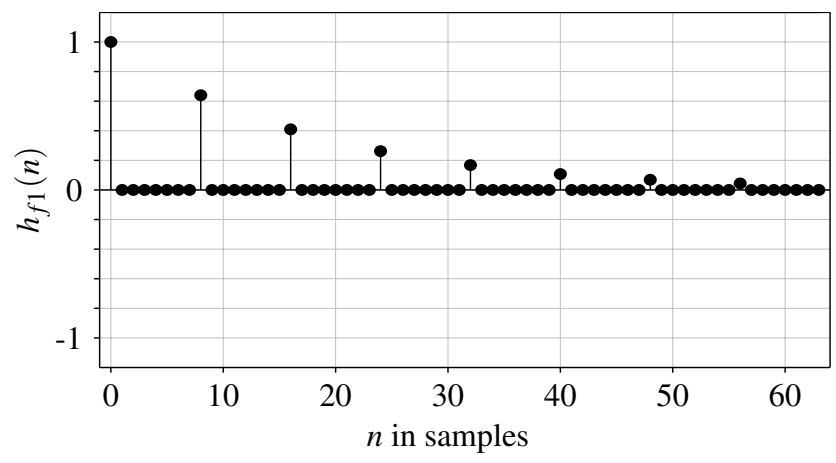

Fig. 6. Computed impulse response $h_{f 1}(n)$.

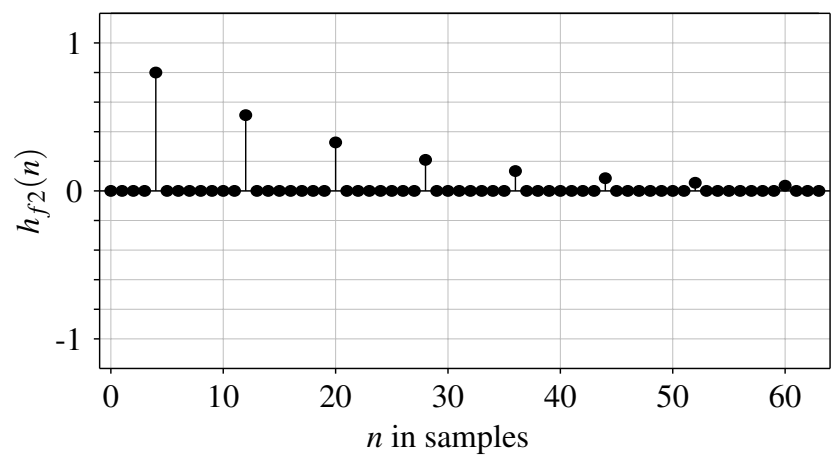

Fig. 7. Computed impulse response $h_{f 2}(n)$. 


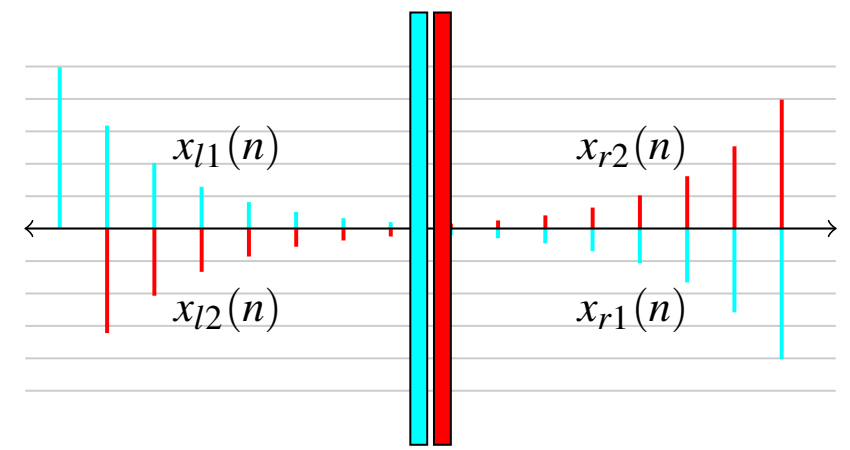

Fig. 8. Signal parts of each loudspeaker after about 70 samples with an unit impulse input signal $x_{\text {in }}(n)=\delta(n)$.

and each impulse decreases by a factor of $g$. This gains an equation for the filter impulse responses

$h_{d}(n)=\sum_{k=0}^{N} g^{2 k} \cdot \delta(n-d \cdot 2 k)$
$h_{t}(n)=\sum_{k=0}^{N} g^{2 k+1} \cdot \delta(n-d(2 k+1))$.

For this example the impulse response is cut after 64 samples. A closer look at $h_{f 1}(n)$ and $h_{f 2}(n)$ explains the behavior of the system. Let us assume a single unit impulse as input signal $x_{\text {in }}(n)=\delta(n)$, which has to be radiated to the radiation direction. The first impulse $h_{f 1}(0)=1$ represents this desired radiation of the untouched signal to the radiation direction. The first impulse at the second radiator $h_{f 2}(d)=g$ gains the needed $180^{\circ}$ phase shifted, attenuated wave to suppress the unwanted backward radiation. Note, that there already is a $180^{\circ}$ phase shift between forward and backward radiated signal of each source due to its dipole character. Since the second radiator itself has a backward radiation, too, a further, again attenuated impulse $h_{f 1}(2 d)=g^{2}$ is needed to keep the desired radiation uninfluenced. The attenuation leads to an exponential decay of needed signal "echoes" to achieve the single sided radiation.

Figure 8 shows the signal parts of the sources after about 70 time steps. This shows the direct dependency of the additionally needed power and the physical attenuation from the origin of the wave to origin of the $180^{\circ}$ phase shifted wave.

\subsection{Stereo radiation}

Besides the single-sided radiation this approach allows the radiation of independent signals to each direction. The suppression of the crosstalk between the directions is equal to the suppression of backward radiation of the single-sided radiation. The stereo radiation is easily achieved by applying the filter unit $\left(h_{f 1}(n), h_{f 2}(n)\right)$ to both input signals $x_{\text {in } 1}(n)$ and $x_{\text {in } 2}(n)$. The filter outputs are summed up by choosing different radiation directions for each filter unit as shown in

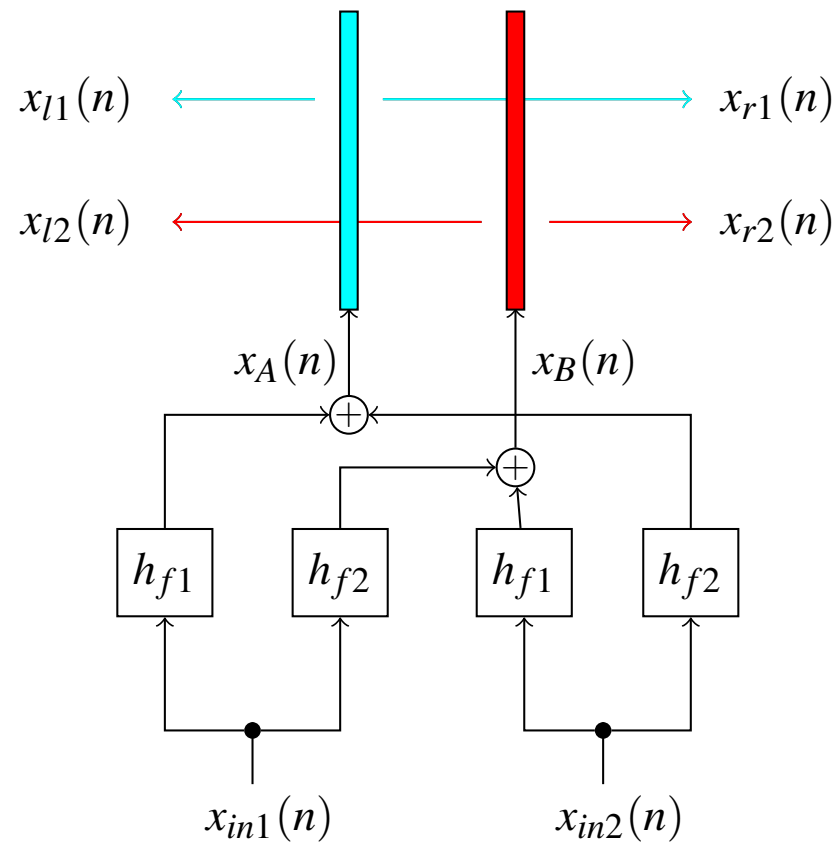

Fig. 9. Scheme of the system with dedicated signal processing for every independent input signal summed up to stereo radiation.

Fig. 9. Now the sum of the output of filter $h_{f 1}(n)$ driven by input signal $x_{\mathrm{in} 1}(n)$ and the output of filter $h_{f 2}(n)$ driven by input signal $x_{\mathrm{n} 2}(n)$ has to actuate panel 1. Panel 2 has to be actuated by the sum of the output of filter $h_{f 1}(n)$ driven by $x_{\mathrm{in} 2}(n)$ and output of filter $h_{f 2}(n)$ driven by $x_{\mathrm{in} 1}(n)$.

$x_{A}(n)=h_{f 1}(n) \cdot x_{\mathrm{in} 1}(n)+h_{f 2}(n) \cdot x_{\mathrm{in} 2}(n)$

$x_{B}(n)=h_{f 1}(n) \cdot x_{\mathrm{in} 2}(n)+h_{f 2}(n) \cdot x_{\mathrm{in} 1}(n)$

Certainly this causes higher restriction to the needed damping factor to avoid clipping (see Sect. 3.3).

\section{Prototype system}

The developed prototype is based on a Texas Instruments floating-point DSP built in the PADK evaluation board of Lyrtech with all needed periphery. The real-time filtering of any input signal for single-sided radiation and stereoradiation, as well as a calibration algorithm to determine the filter impulse responses were implemented. The calibration algorithm makes use of the exponential sine sweep measurement technique. The used length of the filter impulse responses $h_{f 1}(n)$ and $h_{f 2}(n)$ amounts to 512 samples. The sampling frequency was set to $44.1 \mathrm{kHz}$. As loudspeakers the Panphonics S60 audio elements (Pan, 2009) with a size of $600 \times 600 \times 4(w \times h \times d$ in $\mathrm{mm})$ are used. The distance between the loudspeakers is set to $10 \mathrm{~mm}$. The microphone-loudspeaker distance during the automatic calibration is $5 \mathrm{~cm}$. 


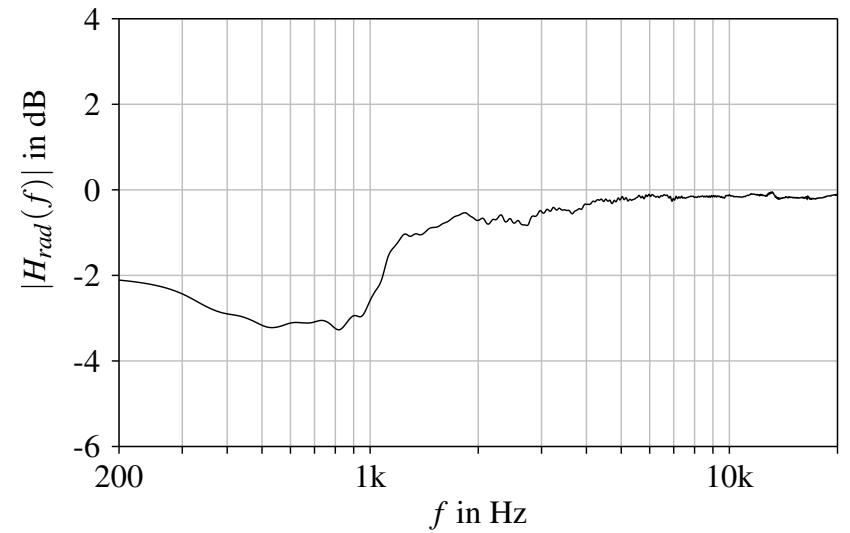

Fig. 10. Difference of the compared frequency responses at the radiation side between enabled and disabled signal processing.

\section{Measurement results}

The measurements are done on axis with microphones on both sides at the same distance of about $20 \mathrm{~cm}$. Once again the exponential sine sweep method is applied to get the impulse responses and transfer functions of the entire system. The reflections of the walls at the measurement location are removed in the computed transfer functions by windowing its impulse responses.

The transfer functions of the entire system are measured twice for enabled and disabled signal processing. That means for the case of disabled signal processing the panel located to the radiation side is actuated, whereas the panel to the suppression side stays passive. The achieved differences between enabled and disabled signal processing at both sides $\left|H_{\text {rad }}(f)\right|$ and $\left|H_{\text {sup }}(f)\right|$ are depicted in Figs. 10 and 11. The behavior on the radiation side of the double dipole with activated signal processing was desired to have low differences to a single radiating dipole loudspeaker.

The measured result in Fig. 10 shows only minor differences between the enabled and disabled signal processing of less than $4 \mathrm{~dB}$. For most audio signals like speech or music the auditory impression approves nearly no perceivable differences when activating the signal processing.

However, the second radiation direction was desired to be suppressed by the system to achieve a single-sided radiation by dipoles. Figure 11 shows the difference of the transfer function for disabled and enabled signal processing of the system. A high broadband active attenuation is observable. For increased frequencies $(>5 \mathrm{kHz})$ the attenuation values show higher fluctuations. Explanations for this behavior could be the used passive amplifiers providing the required bias voltage for the electrostatic panels. For higher frequencies the capacitive behavior of the panels causes a high current. The used combination of the passive adapter and a common hi-fi amplifier is not sufficient to provide accurate signals.

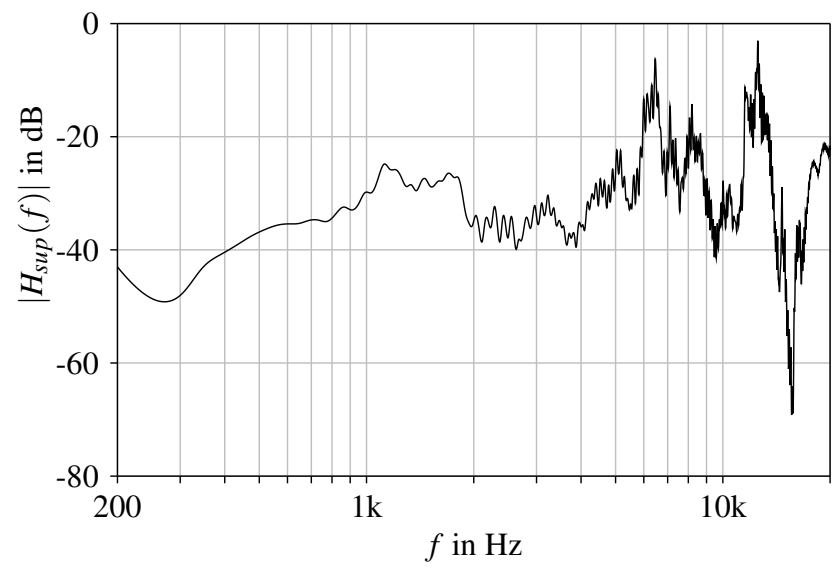

Fig. 11. Difference of the compared frequency responses at the suppression side between enabled and disabled signal processing.

\section{Discussion}

A limitation of this approach is the additional power needed to suppress all occurring "echoes". To avoid quantization clipping the filters have to be normalized. As countermeasure to the digital normalization a higher amplification is needed to get a similar effective output compared to single source radiation. This additional power needed directly depends on the attenuation of radiating through an actuator and requires amplification headroom of the sources for low attenuation values. The decay of the filter impulse response, hence the required filter length, depends on the attenuation as well. The attenuation through a typical actuator is usually sufficient in the audio domain, leading to reasonable ampflications and filter lengths.

In principle the proposed approach of controlling the radiation of plane wave actuators with forward and backward radiation should work independently of the physical domain of the wave. The advantages of this approach compared to passive methods have to be investigated separately for each physical domain. However, it makes use of the cancellation by destructive superposition of waves, hence it is applicable wherever sources of travelling waves radiate into 2 opposite directions.

\section{Conclusion}

The results of the comparative measurements demonstrate the functionality of the developed approach of filter computation for the audio domain. With its practical realization a high broadband active attenuation of nearly $40 \mathrm{~dB}$ is achieved at the suppression side of the double dipole loudspeaker. The influences to the desired radiation side are very low and produce nearly no audible changes. Hence, an effective system was developed that achieves a high active attenuation of the unwanted radiation side without influencing the desired 
radiation direction. This approach of suppressing the second radiation direction of dipole loudspeakers is applicable wherever loudspeakers have to be integrated and no further passive efforts to suppress the backward radiation is wanted or possible.

According to this developed system further applications can be found beyond the single-sided radiation. For example the extension to a stereo radiation of different independent signals to both sides is already realized.

\section{References}

Panphonics Oy, Audio Element Manual v1.1, Panphonics, http: //www.panphonics.com/pdf/AudioElementManualv1-1.pdf, 2009.

Corbach, T., Holters, M., and Zölzer, U.: Flat loudspeaker radiation characteristics controlling method, involves compensating sound signals that are emitted from loudspeaker by phase shifted compensation signal in moment of passage via another loudspeaker, German Patent - DE102007049407A1, 2009.

Farina, A.: Advancements in impulse response measurements by sine sweeps, in: 122nd AES Convention, Vienna, Austria, 2007.

Olson, H. F.: Gradient Loudspeakers, Journal of the AES, 21, 8693, 1973. 